\title{
Die unendliche Diskussion um Blutdruckziele
}

Fragestellung: Welche Blutdruckwerte sollten bei Diabetespatienten unter antihypertensiver Therapie angestrebt und erreicht werden?

Hintergrund: Nachdem die Fachgesellschaften die Empfehlung von sehr strengen Werten $<130 / 80 \mathrm{mmHg}$ korrigiert haben, wird seit Jahren um die Blutdruckziele, v. a. auch bei Diabetikern, gestritten. Häufig bestimmt die jeweils neueste Studie die Debatte (derzeit SPRINT-Studie ohne Diabetiker). An Studien und Metaanalysen mangelt es nicht, nur kommen sie leider zu widersprüchlichen Ergebnissen. Unter neuen Gesichtspunkten wurde daher eine weitere umfangreiche Metaanalyse durchgeführt.

Patienten und Methoden: Berücksichtigt wurden nur Studien mit einer Dauer von mindestens 12 Monaten und mit mindestens 100 Diabetespatienten (überwiegend Typ-2-Diabetes). Sie

\section{Originalie}

Brunström M u. Carlberg B. Effect of antihypertensive treatment at different blood pressure levels in patients with diabetes mellitus: systematic review and meta-analyses. BMJ 2016;352:i717. mussten ein Antihypertensivum gegen Placebo oder Kombinations- gegen Monotherapie vergleichen und bestimmte Blutdruckzielwerte anstreben.

Insgesamt erfüllten 49 Studien mit 73.738 Patienten diese Kriterien. Im Durchschnitt betrug die Studien- dauer 3,7 Jahre und die Blutdruckdifferenz zwischen Kontrollund Interventionsgruppe lag bei 10,2 $\mathrm{mmHg}$.

Ergebnisse: Der Erfolg der antihypertensiven Therapie hängt entscheidend vom Ausgangsblutdruck ab:

- Bei Werten über $150 \mathrm{mmHg}$ kommt es bei einer Senkung um im Mittel $10 \mathrm{mmHg}$ zu signifikanten Abnahmen von Gesamtmortalität, kardiovaskulärer Mortalität, Herzinfarkten, Schlaganfällen und Herzinsuffizienz.

- Schon bei Werten von 140 - 150 mmHg wird der Nutzen bei allen Endpunkten geringer: Nur bei Gesamtmortalität, Herzinfarkt und Herzinsuffizienz bleibt er signifikant, nicht aber bei den anderen Endpunkten.

- Bei Ausgangswerten unter $140 \mathrm{mmHg}$ verringert sich der Benefit der Therapie weiter. Kardiovaskuläre Mortalität und Gesamtmortalität steigen sogar signifikant an.

- Bei Betrachtung der unter Therapie erreichten Werte gilt: Werte von 130 - 140 mmHg senken das Risiko nicht stärker als Werte über $140 \mathrm{mmHg}$. Ein Blutdruck unter $130 \mathrm{mmHg}$ ist tendenziell schlechter (ausgenommen zur Verhinderung von Schlaganfällen).

Schlussfolgerung: Bei Diabetikern liegt die Grenze für die Indikation zur antihypertensiven Behandlung und für den Zielwert bei $140 \mathrm{mmHg}$ systolisch.

\section{- Kommentar Prof. Dr. med. Heinrich Holzgreve}

\section{Spezifische Nebenwirkungen der intensivierten Therapie beachten}

Schon Pilatus fragte: „Was ist Wahrheit"? Offenbar gibt es mehrere Wahrheiten. Denn sowohl die Befürworter als auch die Gegner strenger und konventioneller Blutdruckzielwerte zwischen 130 und 140 mmHg können sich auf die „Evidenz" einer Vielzahl von Studien und Metaanalysen stützen. Die Widersprüche werden meist auf methodische Unterschiede und unerkannte Störfaktoren in den Einzelstudien zurückgeführt. Möglicherweise aber ist die aggressive Therapie selbst eine entscheidende Variable. Denn kürzlich zeigte sich in einer Studie von niedergelassenen Ärzten in England, dass eine intensivierte Blutdrucksenkung unerwartete, spezifische Nachteile

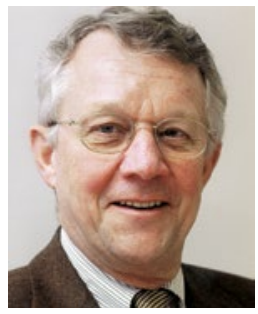

Prof. Dr. med. Heinrich Holzgreve

Internist, Kardiologische Praxis

Burgstr. 7, 80331 München

heinrich.holzgreve@t-online.de hat: Patienten kamen seltener zu Kontrollen, nahmen weniger zuverlässig die Medikamente, machten die Dosissteigerung für Befindlichkeitsstörungen verantwortlich und lehnten weitere Verordnungen ab. Da sticht der graue Alltag die Zuversicht auf Evidenz.

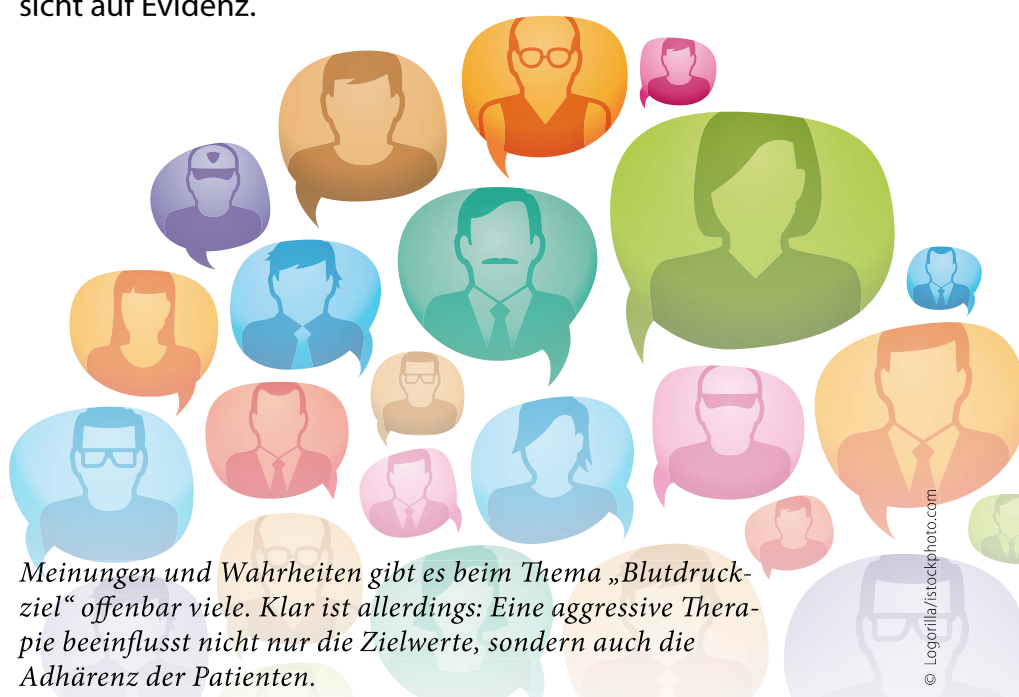

